\title{
Inhibitory Control and Preschoolers' Use of Irregular Past Tense Verbs
}

\author{
Amanda Rose Yuile \\ University of Illinois at Urbana-Champaign \\ Mark A. Sabbagh \\ Queen's University at Kingston
}

\begin{abstract}
We investigated whether children's inhibitory control (IC) is associated with their ability to produce irregular verb forms as well as learn from corrective feedback following over-regularization errors. Forty-eight 3.5 to 4.5 year old children were tested on the irregular past tense and provided with adult corrective input via models of correct use or recasts of errors following ungrammatical responses. IC was assessed with a three-item battery of tasks that required suppressing a prepotent response in favor of a non-canonical one. Results showed that IC was associated with children's initial production of irregular forms, but not associated with their post-feedback production. Findings are discussed in terms of current theories of past-tense use and acquisition.
\end{abstract}

Keywords: grammatical development, past tense, morphology, inhibitory control, language acquisition, preschool-aged children

Insights into language acquisition can come from understanding the processes that contribute to children's production of language errors. For instance, although for many verbs in English the past tense is formed by adding "-ed" to the verb's stem (e.g., walked, picked, kissed), there is a second category of verbs that instead form the past tense by a change in the verb's stem (e.g., run ran) or undergo no change (e.g., put - put). Children spend many years creating a regularized past tense form for a stem that conventionally takes an irregular past-tense form (e.g., saying "falled" instead of "fell"), a phenomenon called "overregularization" (Maratsos, 2000; Maslen et al., 2004). Perhaps most intriguing is that individual children differ in their ability to successfully produce irregular forms (Marcus, 1993). The main goal of the present study was to explore these potential

This is an unedited preprint of an article in press at Journal of Child Language.

Please send correspondence to Amanda Rose Yuile (amandah3@illinois.edu).

We thank the families who participated in this study. This work was funded by an NSERC Discovery Grant (RGPIN250004-11) to M. Sabbagh. We are grateful to members of the Early Experience Laboratory at Queen's for their assistance with data collection and coding. We also thank an anonymous reviewer for providing helpful feedback. This work was presented at the 41 st annual Boston University Conference on Language Development. 
differences in relation to children's developing executive functioning skills, in particular their developing capacity to use cognitive inhibition, or as it is more commonly called, inhibitory control (IC). In doing so we hope to gain insight into how domain-general cognitive skills might support the development of an adult grammar.

IC is a domain-general cognitive skill that allows one to resist a prepotent or habitual way of responding in order to execute an alternative that is appropriate to the given task. A paradigm example of a situation in which IC is required is the Stroop task. In the Stroop task, participants are confronted with a color word (e.g., "green") that is written in a color that is either consistent (e.g., green) or inconsistent (e.g., red) with the meaning of the word. Participants' task is to report the color of the ink. When the color of the ink conflicts with the meaning of the word, participants are typically slower to respond and make more errors. This is because our prepotent response is to report the word rather than the color of the ink. IC is therefore required to suppress the tendency to read the word, and instead correctly report the ink color. Simpler versions of the Stroop task have been created for young children and have shown that the capacity for IC goes through substantial growth over the preschool period (see e.g., Carlson, 2005).

There are several reasons one might expect IC to be associated with irregular past tense production. IC has been linked to a number of linguistic phenomena in which children need to select the most appropriate response from a range of possible alternatives. Notably, cognitive mechanisms including inhibitory control have been associated with children's ability to use linguistic evidence to resolve a syntactic ambiguity in online sentence processing (Woodard et al. (2016)), use context to differentiate between homophones (e.g., knew and new) (Khanna \& Boland, 2010), and resolve referential ambiguity through perspective taking (Nilsen \& Graham, 2009). In the case of resolving a syntactic ambiguity, Woodard, Pozzan, and Trueswell (2016) suggest that executive function skills are engaged when one inhibits their initial interpretation of a sentence in order to interpret the linguistic material in a manner that is consistent with the current task demands. Resolving a lexical ambiguity (i.e., homophone processing) might require suppressing the irrelevant word meaning (Khanna \& Boland, 2010). Thus, inhibitory control processes are engaged during online sentence comprehension to resolve ambiguity by suppressing competitors.

In a similar way, the successful production of irregular past tense verb forms may rely on the suppression of a prepotent tendency to apply regular inflection in favor of the irregular construction. There are two possible sources of a prepotent tendency to apply regular inflection to an irregular verb. According to dual-route theories of past tense production, the past tense is most commonly formed through the automatic or habitual application of an abstract "add -ed" rule (e.g., Marcus et al., 1992; Pinker, 1999; Pinker \& Ullman, 2002). On this account, irregular forms are stored in lexical memory and, when successfully retrieved, block the application of the rule. IC skills akin to those described above may support the blocking of the habitual form and allow children to successfully use the retrieved past tense form. A second possible source of a prepotent tendency to apply regular morphology comes from single-route theories of past tense production, which argue that children average across sentences in their input to generate a pattern before forming abstract linguistic categories. When averaging across input sentences, regular and irregular past tense forms face competition from other phonologically similar forms. Therefore, the successful retrieval of an irregular form (e.g., fly-flew) requires suppressing similar sounding regular forms (e.g., die-died, lie-lied) (Maslen et al., 2004; Tomasello, 2000). On this account, the hypothesis is that inhibitory control may aid in the suppression of competition from phonologically similar forms. Although both models leave room for debate about the origin of overregularization errors (i.e., whether it is because 
of an abstract rule or a statistically-based pattern), each suggests that errors in irregular production may be related in part to failures of IC, and as such, developmental gains in IC may lead to a decrease in overregularlization errors.

Despite its clear importance in theoretical models, the question of whether children's IC skills represent a rate-limiting factor in their successful production of the irregular past tense has received little attention. We know of two prior experiments that consider this question: The first comes from Ibbotson and Kearvell-White (2015) who found that 5-year-old children who performed better at a Sun/Moon Stroop task also performed better at a past tense elicitation task. Though an important first step, the work is preliminary and limited by the use of only one task to characterize inhibitory control skills, a task which required a verbal response. As the authors note, a battery of tasks that encompass a broader range of responses would help strengthen the conclusion that inhibitory control is associated with correct past tense production. Additionally, the reported analyses assessed past tense formation generally, rather than focusing on irregular morphology, thereby making it unclear the extent to which the findings may pertain to theoretical models of past tense production. The second finding comes from Gandolfi and Viterbori (2020) who longitudinally-measured the influence of children's IC skills on their language development. In their study, IC was assessed using a battery of 5 tasks administered when children were 24- to 32-months-old. Using principle component analysis, the authors identified two independent IC sub-components: Response Inhibition (the ability to inhibit a prepotent response) and Interference Suppression (the ability to inhibit conflicting information). Children's receptive morphosyntactic ability was then assessed one year later. Interference Suppression, but not Response Inhibition, was significantly associated with grammatical skills at Time 2 even when age, maternal education, and lexical or grammatical ability at Time 1 were controlled for. These findings suggest that the prior results from Ibbotson and Kearvell-White may be attributable to a more specific Interference Suppression component of IC. However, Gandolfi and Viterbori used a more general measure of grammatical ability, making this interpretation of the prior results only speculative.

Perhaps a more important limitation is that the prior work does not provide direct evidence about why there is a connection between IC and irregular past tense production. As described above, it is possible that because the regular form is prepotent, IC is required to inhibit the tendency to respond with that prepotent form and provide the irregular inflection. An alternative hypothesis is that IC might play an important role in learning irregular past tense forms from input. IC is associated with learning from input in a variety of domains, including theory of mind, language, and mathematics (e.g., Benson et al., 2013; Blair \& Razza, 2007; Bull \& Scerif, 2001; Espy et al., 2004). For instance, IC performance is positively correlated with children's mathematical ability (Bull et al., 1999; Bull \& Scerif, 2001) even afer controlling for age, maternal education, vocabulary, and other cognitive skills (e.g., working memory) (Espy et al., 2004). Here, we suggest that IC may also play a role in promoting uptake of irregular past tense verb forms, just as it promotes learning from input in other domains.

The input that children receive when learning a language can be divided into two general categories. The first is positive evidence, which is any correct utterance spoken in a language. The second is negative evidence, which is information about what is not allowed in a language. Although children do not often receive explicit corrections about the mechanics of language, such as ?no, that is the wrong way to say that,? they are likely to hear a recast - a grammatically correct repetition of their utterance - by the parent in response to the error such as the following (Farrar, 1990; Saxton, 2005; Strapp \& Federico, 2000): 
CHILD: The ball falled down.

MOTHER: The ball fell down.

There is now good evidence that recasts are effective in irregular past tense learning (Chouinard \& Clark, 2003; Proctor-Williams \& Fey, 2007; Saxton, 2000; Saxton et al., 2005; Saxton et al., 1998; Strapp et al., 2008). For example, when recasts are provided in laboratory contexts, children show better learning of irregular past tense forms for novel verb stems than when they are provided only with models of correct use (Saxton et al., 1998).

In the present context there is some reason to believe that IC may be related to learning from recasts because of their unique ability to make salient the errors in the child's speech when children use an incorrect past tense verb form. Parents' recasts provide direct feedback that the child's performance was in error (Clark, 2014; Saxton, 2005), which in turn may catalyze a cascade of well-characterized learning processes that are thought to be related to learning more broadly. Namely, when performance is in error, the ability to monitor the error signal is critical to the ability to revise the basis for performance (see Schulz, 2015). Insofar as overregularization errors reflect at least in part children's erroneous beliefs about the correct past tense form for a given stem, the error signal can cause them to search the alternative form, that the parent provides in the recast. Thus, due to the direct contrast that recasts provide, we hypothesize that recasts promote learning by supporting the process associated with error-related learning. Successful error monitoring may be linked with performance on IC tasks that include performance monitoring and response inhibition. Therefore, one might expect that individual differences in IC would be related to learning from recasts, but not models of correct use.

The current study had two primary aims: (1) to investigate the relationship between IC and children's initial production of irregular past tense verb forms, and (2) to explore whether IC promotes learning irregular past tense verb forms from input. To test this, children watched a prerecorded puppet show designed to elicit overregularization errors of known verbs from children. After the experimenter recorded the participant's initial errors, the pair then re-watched the videos that corresponded to verbs that the child previously overregularized. While talking about the puppet show the second time, the experimenter provided the correct form either just after the participant erred by recasting the error (recast condition) or before the participant made the error by modeling correct use (model condition). Next, children were given a post-feedback production test in which they were asked to describe a picture using a past tense verb form. Finally, children's IC skills were assessed with battery of three tasks (Grass/Snow, Bear/Dragon, and Less is More) that required suppressing a prepotent response in favor of a non-canonical one (e.g., pointing to a white square when the experimenter said ?grass?) without requiring a verbal response from the child. We hypothesized that performance on the EF battery would predict performance on the initial past tense elicitation task and the post-feedback learning outcome measure, such that more advanced IC skills would correlate with a higher rate of correct irregular past tense verb form use in all dependent variable measures. We also hypothesized that IC would interact with the type of input children receive about overregularization errors such that more advanced inhibitory control performance would promote a higher rate of post-feedback irregular past tense production in the recast condition. 


\section{Method}

\section{Participants}

Forty-eight native English-speaking, typically developing children participated in the study. This number of participants was chosen on the basis of a power analysis conducted with G*Power and since then confirmed with the 'pwr' package in R. Our primary research question concerned whether children's IC skills correlated with their initial production of irregular past tense forms and their production of past tense forms following corrective feedback. In line with past work in which IC skills have been associated with other aspects of cognitive development (see e.g., Hughes $\&$ Devine, 2015), we predicted moderate associations $(\rho=.40)$, which would be detected with .80 power given a sample size of approximately 46 . Data collection concluded when our pre-determined sample size $(N=48)$ was reached. Participants were $3 ; 6$ to $4 ; 5$ years old ( $M=46.38$ months, $S D=3.65,27$ females). Participants were recruited from an online participant database including families that had previously participated in studies or were recruited from various locations in the surrounding community area. Participants were from a predominantly European-Canadian, middleclass population reflecting the demographics of the southeast region of Ontario, Canada from which they were drawn (90.8\% White, 7.1\% Visible Minorities, $2.1 \%$ Aboriginal). The average household income in the area is $\$ 89,815 \mathrm{CAD}$. Two participants self-identified as bilingual by informing the experimenter that at least one parent predominantly spoke a language other than English in the home or that they were enrolled in a language immersion school.

This study was approved by the General Ethics Review Board of Queen's University under the project title, "Parent and Child Factors in the Acquisition of Past Tense" and protocol number GPSYC-691-15.

\section{Materials and measures}

\section{IC Measures}

We made use of three standardized behavioral measures designed to assess response-conflict inhibition. The administration and scoring of each task followed established field practice; we refer the reader to the citations below for further task details.

Grass/Snow (Carlson \& Moses, 2001). In the Grass/Snow task, children were asked to point to a white card when the experimenter said "grass" and a green card when the experimenter said "snow." Practice trials were administered until the participant responded correctly on one grass and one snow trial consecutively; participants received feedback on incorrect practice trials. Children passed each test trial if they pointed to the correct colored card for the prompt (i.e., white for grass and green for snow). Practice trials were reverse-scored and combined with the total number of correct test trials to create the participant's total task score (possible range: 0-16).

Bear/Dragon (Reed et al., 1984). In the Bear/Dragon task, children were instructed to follow the directions of a "nice" bear puppet, but not a "naughty" dragon puppet. Practice trials were administered until the participant responded correctly to one bear and one dragon trial consecutively; participants received feedback on incorrect practice trials. Only trials in which the dragon provided a command were scored. Each dragon test trial was given a score of 4 if the child successfully inhibited the commanded action, a score of 3 if the child performed a "strategic movement" (e.g., sitting on hands to avoid performing the action), a score of 2 if the child partially performed the commanded action (e.g., lifted hand to nose and then put it down), a score of 1 if they performed an 
incorrect action, and a score of 0 if they performed the commanded action (possible range: 0-20). A total task score was calculated by summing the number of dragon practice trials administered (reverse-scored) and the scores for all 5 dragon test trials.

Less is More (Carlson et al., 2005). In the Less is More task, children were asked to point to a small reward ( 2 jellybeans) to receive a large award (5 jellybeans). Practice trials were administered until the participant responded correctly (pointed to the smaller reward) and the child expressed explicit understanding of the task rules. For each test trial, children received a score of 2 if they successfully pointed to the smaller reward, a score of 1 if they hovered over the larger amount before pointing to the smaller amount, and a score of 0 if they pointed to the larger reward (possible range: 0-32). A total task score was calculated by summing the total number of practice trials (reverse scored) and the scores for all 16 test trials.

\section{Language Development Measure}

The Peabody Picture Vocabulary Test (PPVT) 4th edition (Dunn \& Dunn, 2007) was used to measure participant's receptive vocabulary development.

\section{Initial Past Tense Elicitation Task}

After completing the PPVT, we tested children's initial production of irregular past tense verb forms. We used a total of 12 real verbs that all take an irregular past tense form: blow/blew, build/built, draw/drew, drink/drank, eat/ate, give/gave, read/read, send/sent, sing/sang, take/took, throw/threw, and write/wrote. In addition, 2 known regular verbs were used to prime the use of regular inflection and ensure that children understood the task. The regular verbs used were show/showed and hug/hugged. Twenty-eight videos were created that depicted puppets acting out 14 different actions (12 irregular verbs, 2 regular verbs; see item list above). There were two corresponding videos for each verb and each was approximately 10-15 seconds long. In other words, there were two trials for each verb.

To begin the task, the experimenter told the child that they were going to watch some videos of puppets acting out everyday actions. She explained to the child that while the videos were playing she would tell them what is happening and when the videos were finished playing the child was to tell her what had just happened. While the videos were playing the experimenter described the action (e.g., "I think he is going to show her a toy. Look! He's showing her a toy!"). At the end of each video, the experimenter prompted production of the past tense by asking children, "What happened?" Within each trial, children were encouraged to produce the verb again when no inflection was provided until they either produced an inflected form or were prompted 3 times. The pair first watched the videos that demonstrated the 2 known regular verbs ( 4 total videos). Children were praised for providing an inflected form during regular verb trials (e.g., "Yeah! Good job!"). Children were required to provide a correct response for at least 3 of the 4 regular verb trials before continuing the irregular verb trials. If a child did not provide a correct response for at least 3 of these trials, they were required to watch all 4 videos again to ensure they understood the task. Only one participant had to re-watch the videos. The experimenter and child then watched 24 videos illustrating 12 irregular verbs. The experimenter recorded participants' responses on a sheet of paper during the task. No feedback was provided during this portion of the task. 


\section{Feedback Task}

Following the initial past tense elicitation task, the experimenter assigned 8 target verbs to either one of two conditions: A model condition or a recast condition. The experimenter semirandomly assigned 4 target verbs to each condition; condition assignment was tracked across participants to ensure that verbs were assigned equally across conditions. For a verb to be assigned to either condition, the participant needed to produce an overregularized form during both trials in the initial elicitation task. Thus, 4 verbs from the previous task would not appear in the feedback task and the exact set of 4 unused verbs varied across participants. In other words, children were only given feedback on verbs that they made overregularization errors on in the initial elicitation task.

The experimenter and child re-watched the videos that corresponded to the 8 target verbs. While these videos were playing the experimenter would describe the action (e.g., "I think he is going to blow up that balloon. Look! He's blowing up the balloon."). After videos depicting verbs in the model condition, the experimenter would model correct use of the irregular past tense form (e.g., "Oh, he just blew up that balloon."), without prompting the child for a response. After videos depicting verbs in the recast condition, the experimenter prompted production of the past tense of each target verb by asking children, "What happened?" The experimenter then recast overregularization errors that occurred in these trials (e.g., If the child said, "He blowed up that balloon." the experimenter responded, "Oh, he blew up that balloon.").

\section{Production Task}

Children's production of the past tense of the 8 target verbs from the feedback task was tested using a probe modelled after Berko's "Wug" test (Berko, 1958). Although this task asks the same question as the initial production task, we chose to change the structure slightly to keep children engaged and entertained throughout the experiment. In this test, children were told that they were going to look at some pictures with the experimenter. There were two pictures per trial and two trials per verb. Children were first shown a picture depicting the target verb, which the experimenter described (e.g., children saw a picture of a girl stacking blocks and the experimenter said, "She's building a tower!"). Children were then shown a second picture depicting the completed action and instructed to finish the experimenter's accompanying sentence (e.g., children saw a picture of a girl with a tower of blocks and the experimenter said, "She just .").

\section{Procedure}

\section{Arrival}

To begin the appointment, a female experimenter explained the study and consent process to the parent; parents were informed of the predictions for the outcomes of the study as a part of the consent process. Then the experimenter engaged the child in playing together with some toys while the parent read and signed the consent forms. Testing began once the parent had completed the consent forms and the child had provided verbal assent.

\section{Testing Session}

Total testing time took approximately 45 minutes. Participants completed the all tasks in the following order: (1) PPVT, (2) initial production task, (3) feedback task, (4) post-feedback pro- 
duction task, and (5) IC task battery. Children were given a t-shirt or a $\$ 10$ gift card to a children's bookstore at the end as a thank you for participating in the study.

\section{Results}

\section{IC and Initial Irregular Past Tense Production}

The first aim of this work was to explore whether children's IC might be associated with their ability to produce irregular past tense verb forms. The 3 IC tasks (Grass/Snow, Bear/Dragon, and Less is More) comprised an internally consistent battery $(\alpha=.79)$ and so a single composite measure was created by averaging the standardized scores from each task for each participant (see Table 1 for task means). The aggregate was significantly correlated with PPVT scores $\left(r_{46}=.33, p=.022\right)$, trending towards significance with age $\left(r_{46}=.26, p=.078\right)$, but not significantly associated with sex, $t_{46}=1.51, p=.137$. These findings are consistent with previous research (Carlson, 2005, see), providing evidence that our IC data is typical.

\section{Table 1}

Descriptive statistics for all measures

\begin{tabular}{lccc}
\hline Variable & $M$ & Range & $S D$ \\
\hline Age (months) & 46.29 & $42-53$ & 3.67 \\
IC & & & \\
$\quad$ Grass/Snow & & & \\
$\quad$ Practice & 2.48 & $2-5$ & .81 \\
$\quad$ Test & 11.69 & $2-16$ & 4.19 \\
Bear/Dragon & & & \\
$\quad$ Practice & 1.28 & $1-4$ & .75 \\
$\quad$ Test & 17.72 & $0-20$ & 4.24 \\
Less is More & & & \\
$\quad$ Practice & 2.21 & $1-4$ & .93 \\
$\quad$ Test & 21.43 & $0-32$ & 8.21 \\
PPVT & 80.79 & $46-116$ & 16.96 \\
Initial Production & 5.75 & $0-17$ & 4.81 \\
Post-test Production & & & \\
$\quad$ Recast & .78 & $0-7$ & 1.48 \\
$\quad$ Model & 1.16 & $0-8$ & 1.76 \\
\hline
\end{tabular}

Each trial in the initial past tense elicitation task was given a score of 1 if the child produced a correct irregular form and a score of 0 if the child produced an uninflected or overregularized form. In the analyses that follow, we use the sum of the score on all trials as our measure of initial irregular past tense production, with a larger score reflecting more irregular form use.

In order to test whether IC was associated with a higher rate of production of correct irregular past tense verb forms, we first examined the correlation between initial irregular past tense production and IC composite scores, and it was found to be moderate and significant $\left(r_{46}=.38, p=.008\right)$. In looking at other possible predictors from our battery of measures, we found that age was also significantly correlated with initial production $\left(r_{46}=.33, p=.021\right)$, 
but PPVT $\left(r_{46}=.26, p=.076\right)$ and sex $\left(t_{46}=-.38, p=.709\right)$ were not (see Table 2 for the full summary of correlations among other predictors). Given this pattern of associations with our outcome variable, we next ran a multiple regression analysis in R (R Core Team, 2013) using the $\operatorname{lm}()$ function from the lme4 package (Bates, Maechler, \& Bolker, 2013); p-values were calculated using Satterthwaite approximation (lmerTest package). Initial production scores were used as the outcome measure and EF and age were included as predictors based on the zero-order correlations reported above. In R syntax the model appeared as follows:

initial production $\sim I C+$ age

This analysis showed that children's initial production of correct irregular past tense verbs was significantly predicted from children's age and IC (see Table 3 for summary of model). Inspection of the model coefficients showed that IC significantly and uniquely predicted children's use of irregular past tense verb forms, but the unique effect of age was smaller and fell just short of standard statistical significance. These results support our main hypothesis that children who scored higher on the IC measures correctly produced irregular past tense verb forms more often than children with lower scores (see Figure 1).

Table 2

Correlations between predictors and initial production

\begin{tabular}{lcccc}
\hline Variable & Sex & Age & PPVT & IC \\
\hline Age & -.13 & & & \\
PPVT & -.06 & .03 & & \\
IC & .22 & .26 & $.33^{*}$ & \\
Initial Production & -.06 & $.33^{*}$ & .26 & $.38^{* *}$ \\
\hline${ }^{*} p<.05,{ }^{* *} p<.01$ & & & &
\end{tabular}

Table 3

Summary of Linear Regression Analysis Predicting Initial Production

\begin{tabular}{lcccc}
\hline Variable & $b$ & $S E$ & $t$ & $p$ \\
\hline Intercept & -9.46 & 8.38 & 1.13 & .265 \\
IC & 1.53 & .67 & 2.28 & .027 \\
Age & .33 & .18 & 1.82 & .075 \\
\hline Note. $R^{2}=.17, F_{2,45}=5.70, p=.006$
\end{tabular}

We excluded PPVT as a predictor in our focal model because it was not significantly correlated our outcome measure. However, as an exploratory analysis, we ran a multiple regression model identical to that reported here, except with PPVT included as an additional predictor. Including PPVT in the model weakened the association between IC and initial production scores, $b=1.25, t_{44}=1.77, p=.085$. However, in this model PPVT failed to account for a unique amount of the variance in initial production scores, $b=.05, t_{44}=1.18, p=.246$. Additionally, we used BIC to evaluate the model fit. The model without a vocabulary parameter had a slightly more parsimonious fit $(\mathrm{BIC}=290.53)$ than the model with vocabulary included $(\mathrm{BIC}=292.91)$. 


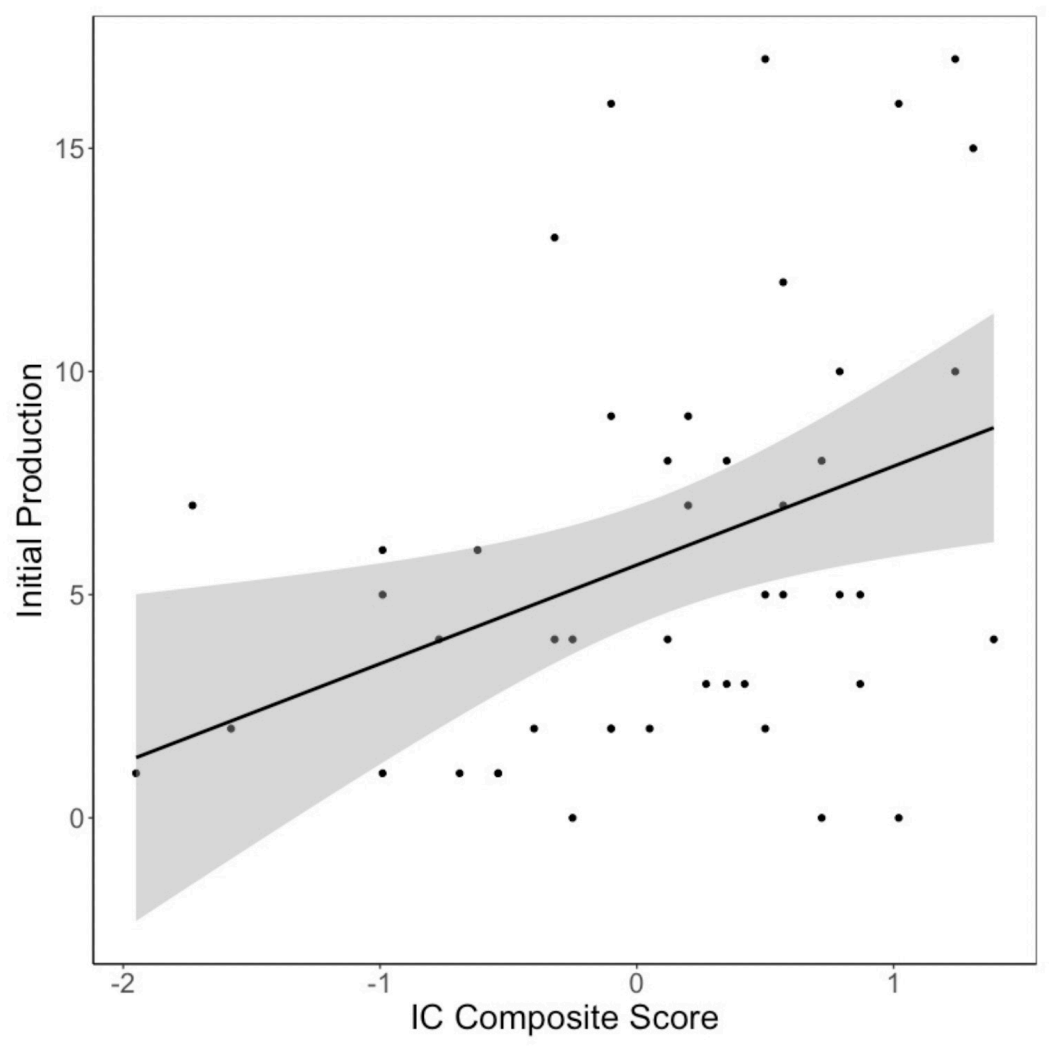

Figure 1

Relation between Initial Production and IC

In summary, we found that children's IC task scores significantly predicted children's production of irregular past tense verb forms. In addition, we found that children's receptive vocabulary was not significantly associated with their ability to produce irregular past tense verb forms in our task. Together, these findings suggest that children's ability to successfully produce an irregular past tense form depends, in part, on their ability to suppress the regular inflection.

\section{IC and Learning Irregular Forms From Input}

The remainder of the analyses includes 37 of the original 48 participants. The reasons for the reduced sample are that eight children did not make overregularization errors during both trials for at least 8 verbs and 3 children chose not to complete the post-feedback production task.

We calculated a production score for each participant by summing across all 16 trials featuring the 8 target verbs in the past tense ( 2 trials per verb), yielding scores potentially ranging from $0-16$. For this task, participants received a score of 0 if they provided an overregularized (e.g., falled) or, an uninflected (e.g. fall) form, and a score of 1 if they provided an irregular form (e.g., fell). Because participants overregularized each target verb during the feedback task, any score larger than 0 represents learning from the feedback.

Previous research comparing negative and positive input in grammar development has found that children revise initial errors at a higher rate following recasts than following models (e.g., Sax- 
ton, 2000). To test whether the same pattern of findings would reproduce in our sample, we conducted a paired-samples $t$-test to compare post-feedback production scores in the model and recast conditions. Contrary to previous results, we did not find that children learned new irregular past tense forms better from recasts relative to models, $t_{36}=1.69, p=.100$.

The second aim of this work was to explore whether IC promotes learning irregular past tense verb forms from input. To test this, we ran a mixed-effects linear regression using the same general methods mentioned above. We used post-feedback production scores as the outcome variable. We included the random intercept of participant; random participant slopes could not be included because IC, age, and vocabulary did not vary within participants. To choose the model that best fit the data, we first fit a model with the most complex effects structure we felt appropriate given the data and then we performed backwards selection using BIC to decide which terms to remove from the model. Our final model included IC, age, PPVT, feedback condition (recast or model), and the interaction between PPVT and feedback condition as fixed effects, and the intercept of subject as a random effect. In R syntax the model looked like the following:

$$
\text { post-feedback production } \sim I C+\text { age }+ \text { ppvt*cond }+(1 \mid \text { subject })
$$

IC did not account for any of the variance in post-feedback production, $b=-.21, t_{33}=$ $-.93, p=.361$. However, age significantly predicted post-feedback production scores, $b=$ $.15, t_{33}=2.54, p=.016$, as did PPVT, $b=.06, t_{33}=3.49, p=.001$. There was a weak effect of condition, $b=1.91, t_{33}=1.80, p=.080$, that did not reach standard significance levels and ran counter to predictions based on prior literature in that children showed some evidence of learning more in the model condition $(M=1.61)$ than the recast condition $(M=.78)$. Finally, the interaction between PPVT and feedback condition was significant, $b=-.03, t_{33}=2.20, p=.034$. To follow-up this significant interaction, we conducted separate analyses for each feedback condition. In these analyses, the main effect of vocabulary was significant in the model condition, $b=.07, t_{33}=3.53, p=.001$, but not in the recast condition, $b=.02, t_{33}=1.51, p=.141$.

Thus, we did not find evidence in support of our hypothesis that IC promotes learning from input in the domain of irregular past tense production, even when accounting for the two categories of input children typically receive about overregularization errors. We did, however, find evidence that preschooler's vocabulary development is correlated with their ability to learn from adult corrective feedback. We will discuss the potential implications of these results below.

\section{Discussion}

This study explored the relation between inhibitory control and the production of irregular past tense verb forms to better understand the factors underlying the success of the system responsible for past tense production. Specifically, we elicited children's use of known irregular past tense forms and provided feedback for overregularization errors in two conditions (recast and model). Children also completed a 3-item IC task battery. We hypothesized first that performance on the inhibition tasks would contribute to children's successful initial production of correct irregular past tense verb forms and second that inhibitory control would contribute to children's ability to learn from an adult's recasts following overregularization errors. We found clear support for the first hypothesis but no evidence for the second. Specifically, more advanced inhibitory control performance was associated with a higher rate of correct irregular past tense production prior to receiving adult 
corrective input, but not after. In addition, we found children's receptive vocabulary scores did not relate to their initial production of irregular past tense verb forms. Ibbotson and Kearvell-White (2015) also failed to observe a relation between correct past tense production and receptive vocabulary. They suggested that children's retreat from overregularization errors is not a purely linguistic process, but involves domain general cognitive mechanisms such as inhibitory control. This claim is further supported by the findings of Gandolfi and Viterbori (2020) that children's Interference Suppression (a sub-component of IC) predicted their grammatical ability one year later. Our failure to find an association between PPVT and initial production provides additional support for this assertion. Finally, we also found an interaction between children's ability to learn from feedback and their vocabulary development such that children with more advanced vocabulary development learned more from models of correct use.

\section{Theoretical implications of the role of IC in past tense learning}

The observed relation between inhibitory control and past tense morphology suggest that children have a prepotent tendency to apply the regular inflection, which must be suppressed in order to successfully produce an irregular past tense form. As noted above, the prepotency of this abstract regular morphology might come from two potential sources. Our study was not designed to provide a basis for distinguishing between these two potential sources. However, we do think that the specifics of our findings may provide some insight, which we speculatively discuss below. Recall that according to dual-system models, the regular "add -ed" rule is applied unless that application is suppressed and supplanted by the retrieval of an irregular form from lexical memory. On connectionist models, overregularization errors are thought to result from lexical competition between phonologically analogous forms before the abstract representation of the regular inflection has been formed. The correct irregular form loses out due to its lack of frequency in the input relative to the frequency of regular past tense analogs. Increasing exposure to the irregular past tense token is thought to be important for strengthening the representation and extinguishing overregularization errors (Maslen et al., 2004; Ramscar, 2002; Ramscar \& Yarlett, 2007).

Although there are theoretical reasons to believe that IC could be involved in either suppression of a prepotent rule application or in negotiating lexical competition, there is much stronger extent evidence for the former relative to the latter. As reviewed earlier, there is strong evidence that IC skills are necessary to overcome habitual or prepotent patterns of responding generally, and specifically in cases of linguistic processing (see Woodard et al., 2016). In contrast, there is not strong evidence to suggest that the negotiation of lexical competition relies on this same sort of cognitive inhibition. That is, we know of no studies that have specifically tied the resolution of lexical competition to the cognitive inhibition aspect of executive function that we measured here. Instead, lexical competition is typically thought to be resolved through retrieval-based, or "lateral," inhibition wherein the processes associated with the retrieval of one word wind up suppressing (inhibiting) the retrieval of the alternative (e.g., Levy \& Anderson, 2002; Magnuson et al., 2007)(see e.g., Levy \& Anderson, 2002; Magnuson, Dixon, Tanenhaus, \& Aslin, 2007). An interesting example of this kind of inhibition is when learners immersed in a second language come to have slowed access to lexical items from the primary language, possibly due to the repetitive retrieval-induced suppression of that language (Levy et al., 2007). There is currently substantial debate about whether the mechanisms involved in retrieval-induced inhibition are similar to those involved in the kind of tasks that we have here, though direct tests of the question have not found an association (e.g., Noreen \& MacLeod, 2015). 
Thus, although both the dual-system and single-system models include a role for "inhibition," only the dual-system model makes a specific prediction about the kind of cognitive inhibitory control that we found is associated with children's initial irregular past-tense production. Future work that better simultaneously explores the connection between cognitive inhibition, retrievalinduced inhibition, and irregular past tense production is necessary to confirm the specific role that different inhibitory processes might play.

\section{The role of different kinds of feedback}

Previous work supported the claim that recasts provide a unique advantage to the language learner, leading to higher rates of post-feedback error-correction than models of correct use (Chouinard \& Clark, 2003; Proctor-Williams \& Fey, 2007; Saxton, 2000, 2005; Saxton et al., 1998; Strapp et al., 2008). In the current study, we did not find evidence for a similar advantage for recasts. Instead, children's performance on irregular past tense production improved in in both feedback conditions. There are a couple of differences between our design and others that may help explain this discrepancy. First, whereas previous studies have commonly employed a between subjects manipulation to examine this difference, we used a within-subjects, unblocked design. In this design, each participant received each type of corrective feedback. If recasts serve to highlight erroneous utterances the child previously made, its possible that children may have been primed to search their speech for errors after hearing a recast and carried this mindset throughout all trials of the task, not only the recast trials. Second, whereas in previous work children were not prompted to produce any form before they heard models, children in our study were prompted to produce the inflected form during our initial production task. In our experiment, children completed the feedback task immediately after the initial production task. Thus, even children in the model condition heard feedback following their errors. As a result of this temporal proximity, it is possible that these utterances, in part, activated an error-monitoring process similar to the advantage posited for recasts.

We did not find evidence that learning from feedback was associated with IC as we had hypothesized. It is possible that IC may constrain aspects of language production, but might not be engaged during this comprehension process. We hypothesized a relation between IC and learning irregular forms from input due to its relation to learning from input in other domains. For instance, in the domain of mathematics, children's executive functioning skills, including inhibition, predict their mathematical ability (Blair \& Razza, 2007). Solving a math problem involves actively reasoning about all of the components, shifting attention to the relevant component(s) of the problem, and inhibiting any response to the most salient component of the problem. In a similar vein, we reasoned that learning irregular forms from recasts might involve the ability to successfully monitor and prevent errors. Although IC was not associated with this learning, it is possible that other aspects of executive functioning may still interact with this specific error-monitoring processing. The current work did not examine individual differences in working memory capacity, for instance; it is possible that learning from feedback is more closely associated with one's ability to keep in mind and actively reason about both the erroneous utterance and the correct, irregular form. This issue highlights an important difference between our initial production task and our feedback task: The initial production task asked children to inhibit a dominant form to produce a less common form. The feedback task required children to simultaneously monitor their erroneous production (in the recast condition) and the correct form provided by the experimenter to notice the contrast between the two forms. These task differences may have contributed to our findings. In particular, the parallel processing involved in the initial production task strongly engaged IC, while the feedback task may 
require a larger role for working memory to monitor/evaluate errors and corrections. Future work is needed to better understand the processes associated with learning from feedback.

However, we did find evidence for an association between learning from feedback and children's vocabulary development. There was a significant interaction between ppvt scores and feedback condition. In addition, exploratory analyses suggested that children that were further along in vocabulary development showed better post-test performance in the model condition. The evidence for this effect was much weaker in the recast condition. We did not predict these findings, so our interpretation of them is only speculative. Yet this result raises the question of whether and how the efficacy of feedback may change with development. Recasts are a more socially contingent piece of evidence for a verb's morphology, whereas models rely on one's ability to track distributional properties of verb morphology in the input. It seems plausible that the weighting of each type of feedback may shift with development. Strapp et al. (2008) observed developmental change in children's ability to learn novel irregular nouns and verbs. In particular, the authors found that 3-year-olds learned more nouns than verbs from recasts than models, while 5-year-olds learned more verbs than nouns from recasts, and 4-year-olds learned both nouns and verbs equally well from both recasts and models. Future work exploring this possibility directly would help to understand the evolving relation between linguistic experience and the correct production of irregular past tense forms.

\section{Limitations}

While this work indicates that inhibitory control is significantly predictive of preschoolers' ability to produce irregular past tense verbs, it is important to acknowledge its limitations. For instance, while we did administer a strong, reliable battery of inhibitory control tasks, we did not measure other executive function factors that may be related to performance on these tasks such as set switching or working memory. Thus, we cannot ensure that the variance attributed to inhibitory control should not instead be attributed to executive function more broadly.

It is also important to comment on the density of our feedback. Although one of our research questions concerned the effects that linguistic experience can on the production of irregular morphology, our training was dense. The feedback task included training on 8 separate verbs with no filler trials. The type of feedback we provided was based on feedback that parents are known to provide, however the density does not reflect naturalistic data (Marcus, 1993; Proctor-Williams $\&$ Fey, 2007). Thus, it is an open question as to whether we might see the predicted associations between IC and learning if feedback was presented at more naturalistic intervals. Similarly, our measure of children's learning was limited to their performance on an immediate post-test, which may lack ecological validity. Important consolidation of learning events may occur in the days or weeks following feedback. For example, Saxton, Backley, and Gallaway (2005) found that recasts, but not various forms of positive evidence, were associated with the use of correct, grammatical forms after a lag of 12 weeks. Perhaps there is an "offline" learning that children experience between exposure to recasts and the next attempt to recreate that or similar utterances. We may have found better evidence for our predicted effects had we used a more ecologically valid measure of learning.

Finally, we tested a relatively narrow age range that captured an age at which children were expected to know the regular "add -ed" rule and make a relatively high degree of overregularization errors. We do not know whether we would find a similar connection between inhibitory control and overregularization errors had we tested younger children. There is some reason to expect that perhaps we would not. Evidence suggests that early in the development of irregular past-tense pro- 
duction, young children have facility with a subset of irregular verbs prior to their having acquired the general rule (Ervin, 1964). Thus, at this early stage, there is likely no prepotent rule that needs to be suppressed in order to produce the correct irregular form. Further work is required to test this particular prediction, which in turn would provide further evidence regarding the mechanisms underlying correct irregular past-tense production at different ages.

\section{Broader Implications}

It is common in both theoretical and applied settings to distinguish between linguistic and domain-general cognitive processes. What these findings suggest is that while on the one hand, overregularization errors are linguistic, these linguistic errors might, at least in some cases, be secondary to deficits in executive functioning. These deficits can arise from developmental immaturity as we evidenced here. Or, these executive deficits might be characteristic of neurodevelopmental conditions. For instance, a defining feature of Williams Syndrome is difficulty producing irregular, but not regular, inflection (e.g., Thomas et al., 2001). There is also now evidence that individuals with Williams Syndrome struggle with tasks that involve inhibition (e.g., Meghini et al., 2010). Research designs like the one that we developed here might be helpful in determining whether there is an inherent connection between the executive function deficits showing in WS and their correct production of irregular past tense forms. In a similar vein, our findings that executive function is critical for correct production of irregular past tense forms raises questions about other populations in which executive function might be affected. For example, there is evidence that executive functioning generally declines with age (e.g., Butler \& Zacks, 2006), and so an interesting question is whether there are concomitant increases in overregularization errors with age as well.

Finally, while we have referred to IC control as a "rate-limiting" factor throughout this paper, it is important to note that inhibitory control has an enabling function in language acquisition as well. In the current work, we suggest that overregularization errors arise as a result of immature IC skills, rather than lack of knowledge about irregular forms. As children grow and their IC matures, inhibitory control will facilitate the use of appropriate past tense forms. Thus, the current findings highlight the interconnected nature of language acquisition and cognitive development.

\section{Conclusion}

This study provides evidence that individual variation in inhibitory control is predictive of grammatical ability with regard to past tense formation. Specifically, we found that IC was positively associated with children's ability to provide irregular inflection. In contrast, we did not find evidence that IC was associated with children's abilities to make use of adult corrective input following the production of an overregularization verb form. These findings suggest that IC is associated with irregular past tense production because IC is an integral part of the cognitive architecture of the past tense production system. Further work is required to better understand both the specificity of the association between inhibitory control and irregular past tense production, as well as the specific mechanisms underlying this association.

\section{References}

Benson, J., Sabbagh, M. A., Carlson, S. M., \& Zelazo, P. D. (2013). Individual differences in executive functioning predict preschoolers' improvement from theory of mind training. Developmental Psychology, 49, 1615-1627. https://doi.org/10.1037/a0031056 
Berko, J. (1958). The child's learning of english morphology. WORD, 14, 150-177. https://doi.org/ 10.180/00437956.1958.11659661

Blair, C., \& Razza, R. P. (2007). Relating effortful control, executive function, and false belief understanding to emerging math and literacy ability in kindergarten. Child Development, 78 , 647-663. https://doi.org/10.1111/j.1467-8624.2007.01019

Bull, R., Johnston, R. S., \& Roy, J. A. (1999). Exploring roles of the visual-spatial sketch pad and central executive in children?s arithmetical skills: Views from cognition and developmental neuropsychology. Developmental Neuropsychology, 15, 421-442. https://doi.org/10.1080/ 87565649909540759

Bull, R., \& Scerif, G. (2001). Executive functioning as a predictor of children?s mathematics ability: Inhibition, switching, and working memory. Developmental Neuropsychology, 19, 273-293. https://doi.org/10.1207/S15326942DN1903 3

Butler, K. M., \& Zacks, R. T. (2006). Age deficits in the control of prepotent responses: Evidence for an inhibitory decline. Psychology and Aging, 21, 638-643. https://doi.org/10.1037/08827974.21.3.638

Carlson, S. M. (2005). Developmentally sensitive measures of executive function in preschool children. Developmental Neuropsychology, 28, 595-616. https:// doi . org / 10 . $1207 /$ s15326942dn2802 3

Carlson, S. M., Davis, A. C., \& Leach, J. G. (2005). Executive function and symbolic representation in preschool children. Psychological Science, 16, 609-616. https://doi.org/10.1111/j.14679280.2005.01583.X

Carlson, S. M., \& Moses, L. J. (2001). Individual differences in inhibitory control and children?s theory of mind. Child Development, 71, 1032-1053. https://doi.org/10.1111/1467-8624. 00333

Chouinard, M. M., \& Clark, E. V. (2003). Adult reformulations of child errors as negative evidence. Journal of Child Language, 30, 637-669. https://doi.org/10.1017/S0305000903005701

Clark, E. V. (2014). Pragmatics in acquisition. Journal of Child Language, 41, 105-116. https://doi. org/10.1017/S0305000914000117

Dunn, L. M., \& Dunn, D. M. (2007). Peabody picture vocabulary test, fourth edition. Minneapolis, MN, NCS Pearson.

Ervin, S. (1964). Imitation in children's language. In E. H. Lenneberg (Ed.), New directions in the study of language (pp. 163-198). Cambridge, MA, MIT Press.

Espy, K. A., Mcdiarmid, M. M., Cwik, M. F., Stalets, M. M., Hamby, A., \& Senn, T. E. (2004). The contribution of executive functions to emergent mathematic skills in preschool children. Developmental Neuropsychology, 26, 465-486. https://doi.org/10.1207/s15326942dn2601 6

Farrar, M. J. (1990). Discourse and the acquisition of grammatical morphemes. Journal of Child Language, 17, 607-624. https://doi.org/10.1017/S0305000900010904

Gandolfi, E., \& Viterbori, P. (2020). Inhibitory control skills and language acquisition in toddlers and preschool children. Language Learning. https://doi.org/10.1111/lang.12388

Hughes, C., \& Devine, R. T. (2015). Individual differences in theory of mind from preschool to adolescence: Achievements and directions. Child Development Perspectives, 9, 149-153. https://doi.org/10.1111/cdep.12124

Ibbotson, P., \& Kearvell-White, J. (2015). Inhibitory control predicts grammatical ability. PLOS One, 10, e0145030. https://doi.org/10.1371/journal.pone.0145030 
Khanna, M. M., \& Boland, J. E. (2010). Childrens use of language context in lexical ambiguity resolution. Quarterly Journal of Experimental Psychology, 63, 160-193. https://doi.org/10. 1080/17470210902866664

Levy, B. J., \& Anderson, M. C. (2002). Inhibitory processes and the control of memory retrieval. Trends in Cognitive Sciences, 6, 299-305. https://doi.org/10.1016/S1364-6613(02)01923-X

Levy, B. J., McVeigh, N. D., Marful, A., \& Anderson, M. C. (2007). The role of retrieval-induced forgetting during second-language acquisition. Psychological Science, 18, 29-34. https:// doi.org/10.1111/j.1467-9280.2007.01844.x

Magnuson, J. S., Dixon, J. A., Tanenhaus, M. K., \& Aslin, R. N. (2007). The dynamics of lexical competition during spoken word recognition. Cognitive Science, 31, 133-156. https://doi. org/10.1080/03640210709336987

Maratsos, M. (2000). More overregularizations after all: New data and discussion on marcus, pinker, ullman, hollander, rosen \& xu. Journal of Child Language, 27, 183-212. https://doi.org/10. 1017/s0305000999004067

Marcus, G. F. (1993). Negative evidence in language acquisition. Cognition, 46, 53-85. https://doi. org/10.1016/0010-0277(93)90022-n

Marcus, G. F., Pinker, S., Ullman, M., Hollander, M., Rosen, T., Xu, F., \& Clahsen, H. (1992). Overregularization in language acquisition. Monographs of the Society for Research in Child Development, 57, 1-178. https://doi.org/10.2307/1166115

Maslen, R. J., Theakston, A. L., Lieven, E. V., \& Tomasello, M. (2004). A dense corpus study of past tense and plural overregularization in english. Journal of Speech, Language, and Hearing Research, 47, 1319-1333. https://doi.org/10.1044/1092-4388(2004/099)

Meghini, D., Addona, F., Costanzzo, F., \& Vicari, S. (2010). Executive functions in individuals with williams syndrome. Journal of Intellectual Disability Research, 54, 418-432. https: //doi.org/10.1111/j.1365-2788.2010.01287.x

Nilsen, E. S., \& Graham, S. A. (2009). The relations between children?s communicative perspectivetaking and executive functioning. Cognitive Psychology, 58, 220-249. https://doi.org/10. 1016/j.cogpsych.2008.07.002

Noreen, S., \& MacLeod, M. D. (2015). What do we really know about cognitive inhibition? task demands and inhibitory effects across a range of memory and behavioral tasks. PLOS One, 10, e0134951. https://doi.org/10.1371/journal.pone.0134951

Pinker, S. (1999). Words and rules. New York, NY, Harper.

Pinker, S., \& Ullman, M. T. (2002). The past and future of the past tense. Trends in Cognitive Sciences, 6, 456-463. https://doi.org/10.1016/s1364-6613(02)01990-3

Proctor-Williams, K., \& Fey, M. E. (2007). Recast density and acquisition of novel irregular past tense verbs. Journal of Speech, Language, and Hearing Research, 50, 1029-1047. https: //doi.org/10.1044/1092-4388(2007/072)

Ramscar, M. (2002). The role of meaning in inflection: Why the past tense does not require a rule. Cognitive Psychology, 45, 45-94. https://doi.org/10.1016/S0010-0285(02)00001-4

Ramscar, M., \& Yarlett, D. (2007). Linguistic self-correction in the absence of feedback: A new approach to the logical problem of language acquisition. Cognitive Science, 31, 927-966. https://doi.org/10.1080/03640210701703576

Reed, M. A., Pien, D. L., \& Rothbart, M. K. (1984). Inhibitory self-control in preschool children. Merrill-Palmer Quarterly, 30, 131-147. 
Saxton, M. (2000). Negative evidence and negative feedback: Immediate effects on the grammaticality of child speech. First Language, 20, 221-252. https : // doi . org/10 . 1177 / 014272370002006001

Saxton, M. (2005). ?recast? in a new light: Insights for practice from typical language studies. Child Language Teaching and Therapy, 21, 23-38. https://doi.org/10.1191/0265659005ct279oa

Saxton, M., Backley, P., \& Gallaway, C. (2005). Negative input for grammatical errors: Effects after a lag of 12 weeks. Journal of Child Language, 32, 643-672. https://doi.org/10.1017/ S0305000905006999

Saxton, M., Kulcsar, B., Marshall, G., \& Rupra, M. (1998). Longer-term effects of corrective input: An experimental approach. Journal of Child Language, 32, 701-721. https://doi.org/10. 1017/s0305000998003559

Schulz, W. (2015). Neuronal reward and decision signals: From theories to data. Physiological Reviews, 95, 853-951. https://doi.org/10.1152/physrev.00023.2014

Strapp, C. M., Bleakney, D. M., \& Helmick, A. L. (2008). Developmental differences in the effects of negative and positive evidence. First Language, 28, 35-43. https://doi.org/10.1177/ 0142723707084840

Strapp, C. M., \& Federico, A. (2000). Imitations and repetitions: What do children say following recasts? First Language, 20, 273-290. https://doi.org/10.1177/014272370002006003

Thomas, M. S. C., Grant, J., Barham, Z., Gsodl, M., Laing, E., Lakusta, L., Tyler, L. K., Grice, S., Paterson, A., \& Karmiloff-Smith, A. (2001). Past tense formation in williams syndrome. Language and Cognitive Processes, 16, 143-176. https://doi.org/10.1080/01690960042000021

Tomasello, M. (2000). First steps toward a usage-based theory of language acquisition. Cognitive Linguistics, 11, 61-82. https://doi.org/10.1515/cogl.2001.012

Woodard, K., Pozzan, L., \& Trueswell, J. C. (2016). Taking your own path: Individual differences in executive function and language processing skills in child learners. Journal of Experimental Child Psychology, 141, 187-209. https://doi.org/10.1016/j.jecp.2015.08.005 